\title{
Closed form solutions of T-stress in plane elasticity crack problems
}

\author{
Y.Z. Chen \\ Laboratory for Nonlinear Mechanics of Continuous Media, Institute of Mechanics, Chinese Academy of Sciences, Beijing, 100080, \\ P.R. China
}

Received 19 May 1998; in revised form 22 October 1998

\begin{abstract}
The T-stress is considered as an important parameter in linear elastic fracture mechanics. In this paper, several closed form solutions of $\mathrm{T}$-stress in plane elasticity crack problems in an infinite plate are investigated using the complex potential theory. In the line crack case, if the applied loading is the remote stress or the concentrated forces, the T-stress can be derived from the basic field. Here, the basic field is defined as the field caused by the applied loading in the infinite plate without the crack. For the circular arc crack, the T-stress can be abstracted from a known solution. For the cusp crack problems, the T-stress can be separated from the obtained stress solution for which the conformal mapping technique is used. (C) 1999 Elsevier Science Ltd. All rights reserved.
\end{abstract}

Keywords: Fracture; Elasticity

\section{Introduction}

The stress distribution in the vicinity of a crack tip was investigated by Williams (1957). In the cylindrical coordinates $(r, \theta)$, the stress components can be expressed by

$$
\left[\begin{array}{cc}
\sigma_{x} & \sigma_{x y} \\
\sigma_{x y} & \sigma_{y}
\end{array}\right]=\frac{K_{1}}{\sqrt{2 \pi r}}\left[\begin{array}{ll}
f_{11}(\theta) & f_{12}(\theta) \\
f_{12}(\theta) & f_{22}(\theta)
\end{array}\right]+\frac{K_{2}}{\sqrt{2 \pi r}}\left[\begin{array}{ll}
g_{11}(\theta) & g_{12}(\theta) \\
g_{12}(\theta) & g_{22}(\theta)
\end{array}\right]+\left[\begin{array}{cc}
T & 0 \\
0 & 0
\end{array}\right]
$$

where the first two terms in the expansion form are singular at the crack tip, $K_{1}$ and $K_{2}$ denote the mode I and mode II stress intensity factors, respectively, and the functions $f_{i j}(\theta), g_{i j}(\theta)$ represent the angular distributions of the crack tip stresses (Williams, 1957). Meantime, the third term is finite and bounded. In the notation of Rice (1974), the third term is denoted as the T-stress and can be regarded as the stress acting parallel to the crack flanks.

Recently, analytical, numerical and experimental studies have attempted to describe fracture in terms of two parameters, i.e. the stress intensity factor and T-stress. Comparatively speaking, the stress 
intensity factor solutions are well investigated in literature, and a few of the T-stress solutions are available. To evaluate the T-stress, the Eshelby technique was used (Kfouri, 1986). Using the weight function method, the idea for evaluating the T-stress was proposed (Chen, 1985, 1997; Sham, 1991). The T-stress in the multiple crack problem was solved (Chen, 1994). Also, the compendium of T-stress solutions in the crack problems was carried out (Sherry et al., 1995). The plane-strain elastic-plastic crack-tip fields were modeled with modified boundary layer formulation based on two parameters, the stress intensity factor and the T-stress (Betegon and Hancock, 1991).

In this paper, we solve the T-stress problems in the infinite plate with: (1) a line crack, (2) a circular arc crack, (3) a symmetric airfoil crack and (4) a symmetric lip crack. The loading condition is the remote stresses $\sigma_{x}^{\infty}, \sigma_{y}^{\infty}, \sigma_{x y}^{\infty}$, or the concentrated forces applied at a prescribed point $z=z_{0}$. By using the complex variable function method in plane elasticity, all the mentioned problems can be solved in closed form.

For the line crack problem, we prove that the T-stress at the crack tip is equal to

$$
T=\sigma_{x(B)}-\sigma_{y(B)}
$$

where $\sigma_{x(B)}, \sigma_{y(B)}$ are the components at the position of crack tip, which are derived from the basic field. For the second to fourth cases, techniques for separating the T-stress from the total stress expressions are suggested.

\section{T-stress for a line crack with concentrated force or remote stress}

In this paper, the complex variable function method in plane elasticity is used to evaluate the T-stress in crack problems (Muskhelishvili, 1953). According to this method, the stresses $\left(\sigma_{x}, \sigma_{y}, \sigma_{x y}\right)$, the displacements $(u, v)$, the resultant force function $(X, Y)$ can be described by the two complex potentials $\phi(z), \psi(z)$ as follows

$$
\begin{gathered}
\sigma_{x}+\sigma_{y}=4 \operatorname{Re} \phi^{\prime}(z) \\
\sigma_{y}-\sigma_{x}+2 i \sigma_{x y}=2\left[\bar{z} \phi^{\prime \prime}(z)+\psi^{\prime}(z)\right] \\
\sigma_{x}-i \sigma_{x y}=2 \operatorname{Re} \phi^{\prime}(z)-\left[\bar{z} \phi^{\prime \prime}(z)+\psi^{\prime}(z)\right] \\
\sigma_{y}+i \sigma_{x y}=2 \operatorname{Re} \phi^{\prime}(z)+\left[\bar{z} \phi^{\prime \prime}(z)+\psi^{\prime}(z)\right] \\
f=-Y+i X=\phi(z)+z \overline{\phi^{\prime}(z)}+\overline{\psi(z)} \\
2 G(u+i v)=\kappa \phi(z)-z \overline{\phi^{\prime}(z)}-\overline{\psi(z)}
\end{gathered}
$$

where $G$ is the shear modulus of elasticity and $\kappa=(3-v) /(1+v)$ for the plane stress problem, $\kappa=3-4 v$ for the plane strain problem, with $v$ being the Poisson's ratio.

Let us consider the single crack problem, in which the concentrated forces $p_{x}, p_{y}$ are applied at the point $z=z_{0}$ (Fig. 1(a)). Clearly, using the superposition principle, the physical field (Fig. 1(a)) can be considered as the sum of the basic field (Fig. 1(b)) and the perturbation field (Fig. 1(c)).

As pointed out previously (Chen, 1994), it is easier to obtain the T-stress by analyzing the stress component in the vicinity of the left crack tip, $z=t^{+}(t \rightarrow a, t<a)$. Obviously, we have 


$$
\sigma_{x}=\sigma_{x 1}+\sigma_{x 2}
$$

where $\sigma_{x}, \sigma_{x 1}, \sigma_{x 2}$ has been indicated in Fig. 1 .

In this case, we have the following solution for the basic field (Fig. 1(b)) (Muskhelishvili, 1953)

$$
\begin{aligned}
& \phi(z)=F \log \left(z-z_{0}\right) \\
& \psi(z)=-\kappa \bar{F} \log \left(z-z_{0}\right)-\frac{F \bar{z}_{0}}{z-z_{0}}, \quad \text { where } F=-\frac{p_{x}+i p_{y}}{2 \pi(\kappa+1)}
\end{aligned}
$$

Therefore, from (4) we obtain

$$
\sigma_{x 1}=T_{1}=\left.\operatorname{Re}\left[2 \phi^{\prime}(z)-\bar{z} \phi^{\prime \prime}(z)-\psi^{\prime}(z)\right]\right|_{z=a} \quad(\text { at the point } z=a)
$$

In addition, in the perturbation field (Fig. 1(c)), the stresses $\sigma_{x}, \sigma_{x y}$ applied on the crack face are denoted by $P(t), Q(t)$, respectively, which in turn are the inverse of the stresses in the basic field. Thus, the stresses applied on the crack face can be expressed by (Chen, 1994)

$$
\left(\sigma_{y}-i \sigma_{x y}\right)^{+}=\left(\sigma_{y}-i \sigma_{x y}\right)^{-}=P(t)-i Q(t)=\left.\left[-2 \operatorname{Re} \phi^{\prime}(z)-z \overline{\phi^{\prime \prime}(z)}-\overline{\psi^{\prime}(z)}\right]\right|_{z=t}, \quad|t| \leqslant a
$$

Particularly, we have proved that (Chen, 1994)

$$
\sigma_{x 2}=-\frac{2 K_{2}}{\sqrt{2 \pi(a-t)}}+T_{2}+O(\sqrt{a-t}),(t \rightarrow a, t<a)
$$

where $K_{2}$ is the stress intensity factor of second mode and

$$
T_{2}=\left.P(t)\right|_{t=a}=\left.\operatorname{Re}\left[-2 \phi^{\prime}(z)-\bar{z} \phi^{\prime \prime}(z)-\psi^{\prime}(z)\right]\right|_{z=a}
$$

Finally, from (7), (9), (11) and (12) the T-stress is obtainable

$$
T=T_{1}+T_{2}=-\left.2 \operatorname{Re}\left[\bar{z} \phi^{\prime \prime}(z)+\psi^{\prime}(z)\right]\right|_{z=a}
$$

By using (3) and (13), the T-stress can be rewritten as

$$
T=\left.\left(\sigma_{x(B)}-\sigma_{y(B)}\right)\right|_{z=a}
$$

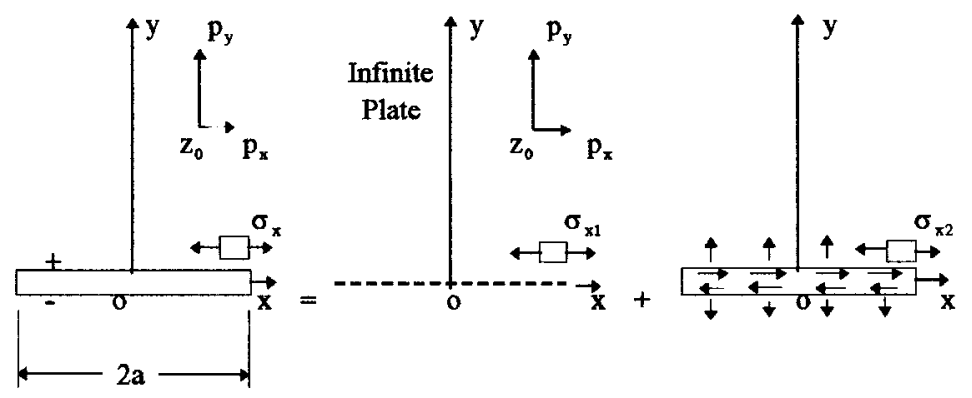

(a)

(b)

(c)

Fig. 1. A single crack with concentrated force loading $p_{x}, p_{y}$, (a) the physical field, (b) the basic field, (c) the perturbation field. 
where the subscript $(B)$ means that, the $\sigma_{x(B)}$, and $\sigma_{y(B)}$ are the stress components in the basic field (Fig. 1(b)). Naturally, the following theorem is proposed.

Theorem: If the single crack in an infinite plate is applied by the concentrated forces $p_{x}, p_{y}$ at the point $z=z_{0}$ (Fig. 1(a)), the T-stress at the left crack tip will be

$$
T=\left.\left(\sigma_{x(B)}-\sigma_{y(B)}\right)\right|_{z=a}
$$

where the components $\sigma_{x(B)}, \sigma_{y(B)}$ are the stresses at the point $z=a$ in the basic field (Fig. 1(b)).

Substituting (8) into (13) yields

$$
T=-2 \operatorname{Re}\left[F \frac{\left(\bar{z}_{0}-a\right)}{\left(z_{0}-a\right)^{2}}+\kappa F \frac{1}{\bar{z}_{0}-a}\right], \quad \text { with } F=-\frac{p_{x}+i p_{y}}{2 \pi(\kappa+1)}
$$

Two particular cases are cited below.

1. If the concentrated forces $p_{x}, p_{y}$ are applied at a point of real axis, i.e. $z_{0}=x_{0}$, from (15) we have

$$
T=\frac{P_{x}}{\pi\left(x_{0}-a\right)}
$$

2. If the concentrated forces $P_{x}, P_{y}$ are applied at the upper side of the left crack tip, $z_{0}=a+i h$ ( $h$-real), from (15) we have

$$
T=\frac{P_{y}}{\pi h}
$$

From above analysis we see that, eqns (13) and (14b) do not depend on the concrete form of complex potentials $\phi(z), \psi(z)$ in the basic field. Therefore, the theorem can also be used for the following cases.

In the second case, it is assumed that a moment $M$ is applied at the point $z=z_{0}$. The complete potentials then take the form (Muskhelishvili, 1953)

$$
\phi(z)=0, \quad \psi(z)=\frac{i M}{2 \pi\left(z-z_{0}\right)}
$$

Substituting (18) into (13) yields

$$
T=\operatorname{Re}\left[\frac{i M}{\pi\left(z_{0}-a\right)^{2}}\right]
$$

In the third case, it is assumed that the remote stresses $\sigma_{x}^{\infty}, \sigma_{y}^{\infty}, \sigma_{x y}^{\infty}$ are applied at infinity. In this case we have the solution for the basic field (Muskhelishvili, 1953)

$$
\phi(z)=\frac{1}{4}\left(\sigma_{x}^{\infty}+\sigma_{y}^{\infty}\right) z, \quad \psi(z)=\left[\frac{1}{2}\left(\sigma_{y}^{\infty}-\sigma_{x}^{\infty}\right)+i \sigma_{x y}^{\infty}\right] z
$$

Substituting (20) into (13) yields

$$
T=\sigma_{x}^{\infty}-\sigma_{y}^{\infty}
$$

This result coincides with that obtained previously (Chen, 1994). 


\section{T-stress in the circular arc crack problem}

For the circular arc crack problem, one may use the coordinates as shown in Fig. 2. It is assumed that the stresses $\sigma_{x}^{\infty}, \sigma_{y}^{\infty}, \sigma_{x y}^{\infty}$ are applied at infinity. In the derivation, we denote

$$
\Psi(z)=\psi^{\prime}(z), \quad \Phi(z)=\phi^{\prime}(z), \quad \Omega(z)=\bar{\Phi}\left(\frac{1}{z}\right)-\frac{1}{z} \bar{\Phi}^{\prime}\left(\frac{1}{z}\right)-\frac{1}{z^{2}} \bar{\Psi}\left(\frac{1}{z}\right)
$$

The obtained solution for the problem is as follows (Muskhelishvili, 1953)

$$
\Phi(z)=F_{1}(z)+F_{2}(z), \quad \Omega(z)=F_{1}(z)-F_{2}(z)
$$

where

$$
F_{1}(z)=\frac{1}{2 X(z)}\left[C_{0} z+C_{1}+\frac{D_{1}}{z}+\frac{D_{2}}{z^{2}}\right], \quad F_{2}(z)=\frac{D_{0}}{2}+\frac{\bar{\Gamma}_{1}}{2 z^{2}}
$$

and

$$
\begin{aligned}
& C_{0}=\frac{1}{2}\left(\Gamma_{1}-\bar{\Gamma}_{1}\right) \sin ^{2}(\alpha / 2)+\frac{4 \Gamma+\left(\Gamma_{1}+\bar{\Gamma}_{1}\right) \sin ^{2}(\alpha / 2) \cos ^{2}(\alpha / 2)}{2\left(1+\sin ^{2}(\alpha / 2)\right)}, \quad C_{1}=-C_{0} \cos \alpha \\
& D_{0}=2 \Gamma-C_{0}, \quad D_{1}=-\bar{\Gamma}_{1} \cos \alpha, \quad D_{2}=\bar{\Gamma}_{1} \\
& \Gamma=\frac{1}{4}\left(\sigma_{x}^{\infty}+\sigma_{y}^{\infty}\right), \quad \Gamma_{1}=\frac{1}{2}\left(\sigma_{y}^{\infty}-\sigma_{x}^{\infty}\right)+i \sigma_{x y}^{\infty} \\
& X(z)=\sqrt{(z-\exp (-i \alpha))(z-\exp (i \alpha))}, \quad\left(\text { taking the branch } \lim _{z \rightarrow \infty} x(z) / z=1\right)
\end{aligned}
$$

Clearly, we have

$$
\sigma_{r}+\sigma_{\theta}=\sigma_{x}+\sigma_{y}=4 \operatorname{Re} \Phi(z)=4 \operatorname{Re} F_{1}(z)+4 \operatorname{Re} F_{2}(z)
$$

After using the translation and rotation transform of coordinates, the counterpart of the complex

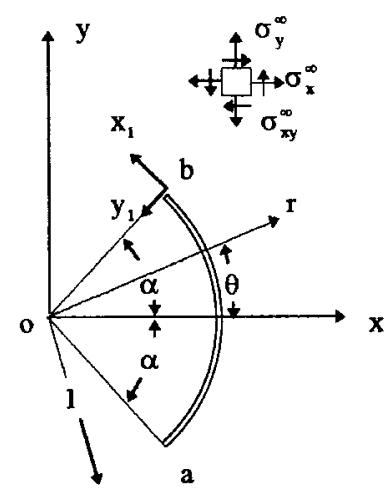

Fig. 2. A circular arc crack. 
potential $F_{1}(z)$ will be denoted by $G_{1}\left(z_{1}\right)\left(z_{1}=x_{1}+i y_{1}\right)$. Also, in the vicinity of $z_{1}=0$ (or in the vicinity of $z=\exp (i \alpha))$ in Fig. 2, $G_{1}\left(z_{1}\right)$ takes the form

$$
G_{1}\left(z_{1}\right)=d_{1} z_{1}^{-1 / 2}+d_{2} z_{1}^{1 / 2}+d_{3} z_{1}^{3 / 2}+\cdots \quad\left(z_{1}=x_{1}+i y_{1}\right)
$$

That is to say, the complex potential $F_{1}(z)$ has no contribution to the T-stress.

Noting the property that $\sigma_{r}=0$ along the circular arc crack face (Fig. 2), therefore, from (26) the T-stress at the crack tip $b(z=\exp (i \alpha))$ will be

$$
T_{b}=4 \operatorname{Re} F_{2}(\exp (i \alpha))=2 \operatorname{Re}\left(D_{0}+\bar{\Gamma}_{1} \exp (-2 i \alpha)\right)
$$

Similarly, from (26) the T-stress at the crack tip $a(z=\exp (i \alpha))$ in Fig. 2 will be

$$
T_{a}=2 \operatorname{Re}\left(D_{0}+\bar{\Gamma}_{1} \exp (2 i \alpha)\right)
$$

Substituting $\Gamma$ and $\Gamma_{1}$ from (25), one may write the T-stress in the explicit form

$$
\begin{aligned}
& T_{b} \\
& T_{a}
\end{aligned}=\sigma_{x}^{\infty}\left[-\cos (2 \alpha)+\frac{(1-\cos \alpha)(3+\cos \alpha)}{2(3-\cos \alpha)}\right]+\sigma_{y}^{\infty}\left[\cos (2 \alpha)+\frac{(1-\cos \alpha)^{2}}{2(3-\cos \alpha)}\right] \mp 2 \sigma_{x y}^{\infty} \sin (2 \alpha)
$$

Clearly, letting $\alpha \rightarrow 0$ in (30), the T-stress for the single-line crack problem is obtainable

$$
\left.T_{a}\right|_{\alpha=0}=\left.T_{b}\right|_{\alpha=0}=\sigma_{y}^{\infty}-\sigma_{x}^{\infty}
$$

\section{T-stress in the cusp crack problems}

The T-stress problem for a symmetric airfoil crack is considered below (Fig. 3). In the problem the remote stresses $\sigma_{x}^{\infty}$ and $\sigma_{y}^{\infty}$ are applied at infinity. In this case, the following mapping function is used (Wu, 1982)

$$
z=\omega(\zeta)=\zeta+\frac{(1-m)^{2}}{\zeta-m} \quad(0<m<1)
$$

which maps the unit circle and its exterior region (in the $\zeta$-plane) into the airfoil crack and its exterior region (in the $z$-plane) (see Fig. 3).

In the following analysis, the complex potentials $\phi(z), \psi(z)$ previously used are rewritten as $\phi_{1}(z), \psi_{1}(z)$. In addition, we let

$$
\phi(\zeta)=\phi_{1}(\omega(\zeta)), \quad \psi(\zeta)=\psi_{1}(\omega(\zeta))
$$

Also, from (3) we can obtain

$$
\sigma_{x}+\sigma_{y}=4 \operatorname{Re} \phi_{1}^{\prime}(z)=4 \operatorname{Re}\left(\phi^{\prime}(\zeta) / \omega^{\prime}(\zeta)\right)
$$

From eqn (5), the traction-free condition along the crack face can be expressed as

$$
\overline{\phi(\zeta)}+\frac{\bar{\omega}(1 / \zeta)}{\omega^{\prime}(\zeta)} \phi^{\prime}(\zeta)+\psi(\zeta)=0 \quad(\zeta \in C, C-\text { the unit circle })
$$



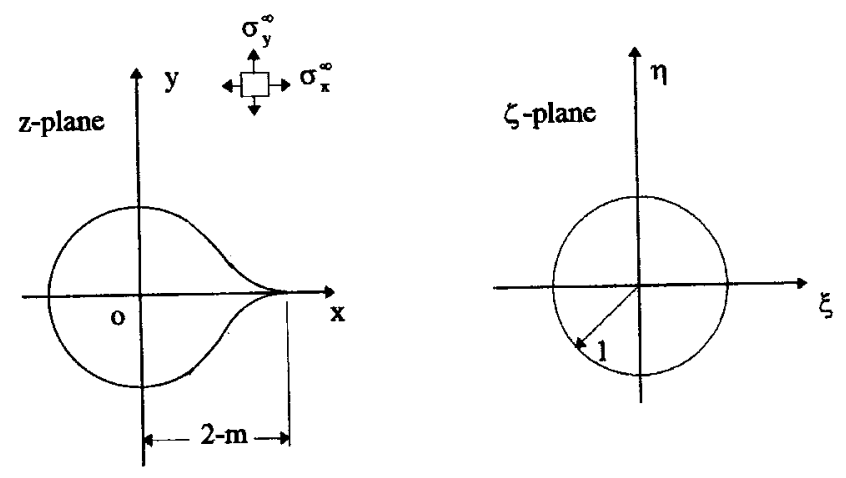

Fig. 3. A symmetric airfoil cusp crack.

Continuation of the complex variable function will give the following solution (Wu, 1982; Chen, 1984)

$$
\phi(\zeta)=\Gamma \zeta-\frac{\left(1-m^{2}\right)^{2}}{1+2 m} \frac{H}{\zeta-m}+\Gamma_{1} \frac{1}{\zeta}, \quad \psi(\zeta)=-\bar{\phi}\left(\frac{1}{\zeta}\right)-\frac{\bar{\omega}(1 / \zeta)}{\omega^{\prime}(\zeta)} \phi^{\prime}(\zeta)
$$

where

$$
H=\phi^{\prime}\left(\frac{1}{m}\right)=\frac{1+2 m}{1+2 m-m^{2}}\left(\Gamma+m^{2} \Gamma_{1}\right), \quad \Gamma=\frac{1}{4}\left(\sigma_{x}^{\infty}+\sigma_{y}^{\infty}\right), \quad \Gamma_{1}=\frac{1}{2}\left(\sigma_{y}^{\infty}-\sigma_{x}^{\infty}\right)
$$

From the singular behavior of the stress component, in the vicinity of crack tip the complex potential $\phi_{1}^{\prime}(z)$ can be expressed as

$$
\phi_{1}^{\prime}(z)=\frac{1}{q}\left(a_{0}+a_{1} q+a_{2} q^{2}+\cdots\right) \quad(q=q(z))
$$

where

$$
q=q(z)=\sqrt{z-(2-m)}
$$

In (38), $a_{0} / q$ is a singular term when $z \rightarrow 2-m$. Since the cusp crack face is traction free, from (34) and (38), it yields

$$
T=4 \operatorname{Re}\left(a_{1}\right)
$$

and from (38) we see that

$$
a_{1}=\left.\frac{\mathrm{d}}{\mathrm{d} q}\left\{q \phi_{1}^{\prime}(z)\right\}\right|_{z=2-m}
$$

Substituting (32) into (39) yields

$$
Q(\zeta)=q(\omega(\zeta))=\frac{\zeta-1}{\sqrt{\zeta-m}}
$$

After considering $\phi_{1}^{\prime}(z)=\phi^{\prime}(\zeta) / \omega^{\prime}(\zeta)$, from (41) we finally obtain 


$$
a_{1}=\left.\left\{\frac{\mathrm{d}}{\mathrm{d} \zeta}\left[Q(\zeta) \frac{\phi^{\prime}(\zeta)}{\omega^{\prime}(\zeta)}\right] / \frac{\mathrm{d} Q(\zeta)}{\mathrm{d} \zeta}\right\}\right|_{\zeta=1}
$$

Substituting (36), (42) into (43), and using (40), T-stress is obtainable

$$
T=-\frac{4 m^{2}}{1+2 m-m^{2}} \Gamma-2(1-m)^{2}+\left(\frac{2 m^{4}}{1+2 m-m^{2}}\right) \Gamma_{1}
$$

or

$$
T=\left(\frac{1-5 m^{2}+4 m^{3}+m^{4}}{1+2 m-m^{2}}\right) \sigma_{x}^{\infty}-\left(\frac{1-3 m^{2}+4 m^{3}+m^{4}}{1+2 m-m^{2}}\right) \sigma_{y}^{\infty}
$$

In the second case, the symmetric lip crack is considered (Fig. 4), and the mapping function is

$$
z=\omega(\zeta)=\zeta-\frac{m^{2}}{\zeta}+\frac{\left(1-m^{2}\right)^{2}}{2}\left(\frac{1}{\zeta-m}+\frac{1}{\zeta+m}\right) \quad(0<m<1)
$$

The mapping relation has been indicated in Fig. 4, and eqns (33)-(35) are still used in this case. The applied remote stresses are denoted by $\sigma_{x}^{\infty}, \sigma_{y}^{\infty}$. As before, the continuation of the complex variable function will give the following solution (Wu, 1982; Chen, 1984)

$$
\phi(\zeta)=\Gamma \zeta+S\left(\frac{1}{\zeta-m}+\frac{1}{\zeta+m}\right)+\left(m^{2} \Gamma-\Gamma_{1}\right) \frac{1}{\zeta}, \quad \psi(\zeta)=-\bar{\phi}\left(\frac{1}{\zeta}\right)-\frac{\bar{\omega}(1 / \zeta)}{\omega^{\prime}(\zeta)} \phi^{\prime}(\zeta)
$$

where

$$
\mathrm{S}=-\frac{\left(1-m^{4}\right)^{2}}{2\left(1+m^{4}\right)\left(1+m^{2}+m^{4}\right)} \phi^{\prime}\left(\frac{1}{m}\right)=-\frac{\left(1-m^{4}\right)^{2}}{2\left(1+m^{4}\right)^{2}}\left[\left(1-m^{4}\right) \Gamma+m^{2} \Gamma_{1}\right]
$$

Similarly, we have

$$
q(z)=\sqrt{z-2\left(1-m^{2}\right)}
$$
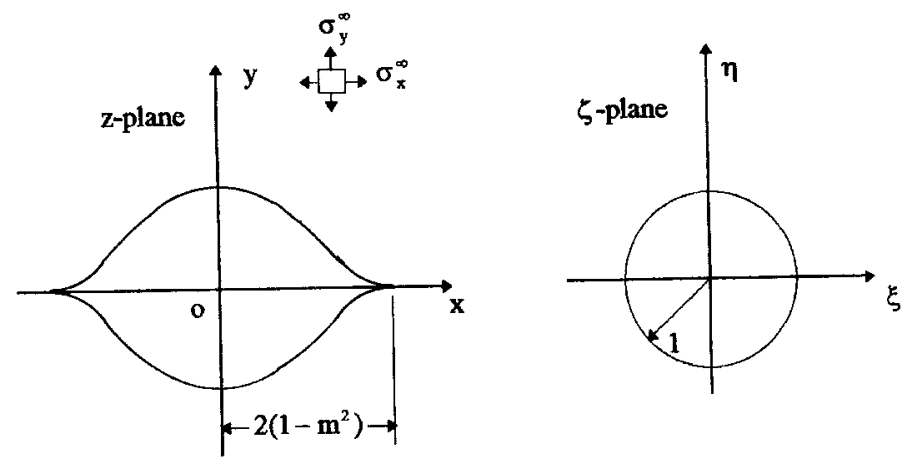

Fig. 4. A symmetric lip cusp crack. 


$$
Q(\zeta)=q(\omega(\zeta))=\frac{(\zeta-1)\left(\zeta+m^{2}\right)}{\sqrt{\zeta\left(\zeta^{2}-m^{2}\right)}}
$$

Clearly, Eqns (38), (40), (41) and (43) are still valid. Finally, from (40) and (43), the T-stress is obtainable

$$
T=\frac{2}{\left(1+m^{2}\right)^{3}}\left(d_{1} \Gamma+d_{2} \Gamma_{1}\right)
$$

where

$$
\begin{aligned}
& d_{1}=\left(1-m^{2}\right)\left(1+6 m^{2}+m^{4}\right)-\frac{\left(1-m^{2}\right)\left(1+m^{2}\right)^{6}}{\left(1+m^{4}\right)^{2}} \\
& d_{2}=-1+4 m^{2}+2 m^{4}-\frac{m^{2}\left(1+m^{2}\right)^{5}}{\left(1+m^{4}\right)^{2}}
\end{aligned}
$$

\section{Remarks}

In the above mentioned examples, from the line crack case, the circular arc crack case to the cusp crack cases, the solution techniques for evaluating the T-stress are not exactly the same. However, from the present analysis we can conclude that, if the closed form solution for the relevant problems can be derived, the T-stress can be evaluated immediately.

\section{Acknowledgement}

This research project is supported by the National Natural Fund of China. I am most grateful to the reviewer for editing the manuscript.

\section{References}

Betegon, C., Hancock, J.W., 1991. Two parameter characterization of elastic-plastic crack-tip fields. ASME J. Appl. Mech. 58, 104-110.

Chen, Y.Z., 1984. Elastic analysis of an infinite plate containing hole with cusps and applied by concentrated forces. Engng Fract. Mech. 20, 573-582.

Chen, Y.Z., 1985. New path independent integrals in linear elastic fracture mechanics. Engng Fract. Mech. 22, 673-686.

Chen, Y.Z., 1994. T-stress in multiple crack problem for an infinite plate. Engng Fract. Mech. 48, 641-647.

Chen, Y.Z., 1997. Novel weight function approach for evaluating T-stress in plane elasticity crack problem. Int. J. Fract. 85, L35-40.

Kfouri, A.P., 1986. Some evaluations of the elastic T-term using Eshelby's method. Int. J. Fract. 30, 301-315.

Muskhelishvili, N.I., 1953. Some Basic Problems of Mathematical Theory of Elasticity. Noordhoff, Groningen.

Rice, J.R., 1974. Limitations to the small scale yielding approximation of elastic-plastic crack-tip fields. J. Mech. Phys. Solids. 22, $17-26$.

Sham, T.L., 1991. The determination of the elastic T-term using higher order weight functions. Int. J. Fract. 48, 81-102.

Sherry, A.H., France, C.C., Goldthorpe, M.R., 1995. Compendium of T-stress solutions for two and three dimensional cracked geometries. Fatigue Fract. Engng Mater. Struct. 18, 141-155.

Williams, M.L., 1957. On the stress distribution at the base of a stationary crack. ASME J. Appl. Mech. 24, 111-114.

Wu, C.H., 1982. Unconventional crack, Part 1: symmetric variation of a straight crack. ASME J. Appl. Mech. 49, $62^{`}-68$. 